\title{
Reliable Guaranteed Cost Control of Uncertain Systems with Nonlinear Perturbations
}

\author{
Nana Wang, Nan Xie* \\ College of Computer Science and Technology, Shandong University of Technology, Zibo, China \\ Email address: \\ 1748462348@qq.com (Nana Wang),xienan@sdut.edu.cn (Nan Xie) \\ ${ }^{*}$ Corresponding author
}

To cite this article:

Nana Wang, Nan Xie. Reliable Guaranteed Cost Control of Uncertain Systems with Nonlinear Perturbations. Advances in Applied Sciences. Vol. 5, No. 4, 2020, pp. 103-111. doi: 10.11648/j.aas.20200504.12

Received: October 10, 2020; Accepted: October 23, 2020; Published: November 4, 2020

\begin{abstract}
Network control system (NCS) is a distributed real-time feedback control system with the continuous development of network technology. It is generally composed of network, controller, actuator and sensor. It has brought great convenience to people in many fields, but it also has many problems, which makes the research of control system more complicated. Recently, there have been some efforts to tackle the reliable guaranteed cost controller design problem, and some good results have also been obtained for the continuous-time and for the discrete-time. However, there have been few results in the literature of an investigation for the reliable guaranteed cost controller design of nonlinear uncertain systems with time-varying state delay and actuator failure. This paper concerns the reliable guaranteed cost control problem of uncertain systems with time-varying state delay and nonlinear perturbations for a given quadratic cost function. The problem is to design a reliable guaranteed cost state feedback control law which can tolerate actuator failures, such that the closed-loop system is asymptotically stable and the closed-loop cost function value is not more than a specified upper bound. Firstly, the existence condition of reliable guaranteed cost control law is given by constructing Lyapunov stability function and using linear matrix inequality (LMI). Secondly, the design method of the optimal reliable guaranteed cost controller is given by solving the convex optimization problem with LMI constraints, which minimizes the upper bound of guaranteed cost for closed-loop systems. In the end, the numerical simulation result illustrates the effectiveness of the proposed method.
\end{abstract}

Keywords: Reliable Control, Guaranteed Cost Control, Time-delay, Actuator Failure, LMI

\section{Introduction}

The problem of designing robust controllers for systems with parameter uncertainties has drawn considerable attention in recent control system literatures. It is also desirable to design a control system which is not only stable but also guarantees an adequate level of performance. One approach to this problem is the so-called guaranteed cost control approach [1]. This approach has the advantage of providing an upper bound on a given performance index and thus the system performance degradation incurred by the uncertainties is guaranteed to be less than this bound. Based on this idea, some significant results have been proposed for the continuous-time case $[2,3]$ and for the discrete-time case [4]. Kao Y. designed a non-fragile $\mathrm{H} \infty$ guaranteed cost controller for uncertain long time delay nonlinear network control system with perturbation of controller parameters [5]. Yin combined the robust control theory to model the NCS, introduced performance indicators, established the internal relationship with network factors, and it also reduced the conservativeness of the results [6]. The controller design for Markov time-delay NCS has also achieved good results, and a design method to maintain NCS performance by switching feedback gain is proposed [7].

In practical application, actuators are very important in transforming the controller output to the plant. Actuator failures may be encountered sometimes. Furthermore, how to preserve the closed-loop system performance in the case of actuator failures will be tougher and more meaningful. Recently, there have been some efforts to tackle the reliable guaranteed cost controller design problem [8-10]. Yao took the uncertain nonlinear time-delay system as the controlled object, and designed a reliable guaranteed cost controller considering the actuator failure of the NCS [11-12]. Zhang J.-S. presented a guaranteed cost control method for multiple time delays and 
actuator failures [13]. Zhu considered the simultaneous failure of the sensor and controller, and modeled as two time-varying and bounded parameters [14]. Sun first attempted to implement $\mathrm{H} \infty$ guaranteed cost control for switched T-S fuzzy stochastic systems with intermittent actuator [15]. For the same fault model, Zou redesigned the static output feedback controller, so that the system can keep stable and satisfy the original performance indexes no matter the actuator fails or not [16].

In this paper, the problem of reliable guaranteed cost control of uncertain systems with time-varying state delay and nonlinear perturbations is considered. In Section 2, the problem under consideration and some preliminaries are given. In section 3, several stability criteria for the existence of the reliable guaranteed cost controller are derived in terms of LMI, and their solutions provide a parameterized representation of the controller. A numerical example is given in Section 4. Finally, Section 5 concludes the paper.

\section{Problem Statement}

Consider the following uncertain systems with time-varying delay and nonlinear perturbations

$$
\begin{aligned}
& \dot{x}(t)=A(t) x(t)+A_{d}(t) x(t-\tau(t))+B(t) u(t)+f(t, x(t), x(t-\tau(t))), \quad t \in R^{+}, \\
& x(t)=\phi(t), \quad t \in[-h, 0],
\end{aligned}
$$

where $\quad x(t) \in R^{n} \quad$ is the state vector, $u(t)=\left[\begin{array}{llll}u_{1}(t) & u_{2}(t) & \cdots & u_{m}(t)\end{array}\right]^{T} \in R^{m}$ is the control vector, $f(\cdot): R_{+} \times R^{n} \rightarrow R^{n}$ is the nonlinear uncertainties, being denoted $f$ in the following. $\tau(t)$ is the time-varying bounded delay satisfying $0 \leq \tau(t) \leq h<\infty, \quad \dot{\tau}(t) \leq d<1$, with $h, d$ being known. $A(t), A_{d}(t)$ and $B(t)$ are matrix functions with time-varying uncertainties, that is

$$
A(t)=A+\Delta A(t), A_{d}(t)=A_{d}+\Delta A_{d}(t), B(t)=B+\Delta B(t),
$$

where $A, A_{d}$ and $B$ are known real constant matrices of appropriate dimensions, $\Delta A(t), \Delta A_{d}(t)$ and $\Delta B(t)$ are uncertain matrices. $\varphi_{\text {is a }}$ given continuous vector-valued initial function on $[-h, 0]$.

Assumption 1. The parameter uncertainties considered are assumed to be bounded and of the form

$$
\left[\Delta A(t) \quad \Delta B(t) \quad \Delta A_{d}(t)\right]=D F(t)\left[\begin{array}{lll}
E_{1} & E_{2} & E_{d}
\end{array}\right],
$$

where $F(t) \in R^{i \times j}$ is an unknown matrix satisfying $F^{T}(t) F(t) \leq I, E_{1}, E_{2}, E_{d}, D$ are known constant matrices of appropriate dimensions describing the structure of uncertainties.

Assumption 2. The nonlinear uncertainty $f$ satisfies

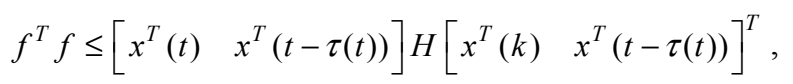

where $H$ is a known constant matrix satisfying

$$
H=\text { Block-diag }\left\{H_{1}^{T} H_{1}, H_{2}^{T} H_{2}\right\}>0 .
$$

Associated with this system is the cost function

$$
J=\int_{0}^{\infty}\left(x^{T}(t) Q x(t)+u^{T}(t) R u(t)\right) d t,
$$

Where $Q$ and $R$ are given positive-definite matrices.

For the control input $u_{i}(t), i=1,2, \cdots, m$, let $u_{i}^{F}(t)$ denote the signal from the actuator that has failed. The following failure model is adopted in this paper:

$$
u_{i}^{F}(t)=\alpha_{i} u_{i}(t), \quad i=1,2, \cdots, m
$$

where

$$
0 \leq \widehat{\alpha}_{i} \leq \alpha_{i} \leq \breve{\alpha}_{i}, \quad i=1,2, \cdots, m
$$

with $\widehat{\alpha}_{i} \leq 1$ and $\breve{\alpha}_{i} \geq 1$.

In the above model of actuator failure, if $\widehat{\alpha}_{i}=\breve{\alpha}_{i}$, then it corresponds to the normal case $u_{i}^{F}(t)=u_{i}(t)$. When $\breve{\alpha}_{i}=0$, it covers the outage case. If $\hat{\alpha}_{i}>0$, it corresponds to the partial failure case, namely, partial degradation of the actuator.

Denote

$$
\begin{aligned}
& u^{F}(t)=\left[\begin{array}{llll}
u_{1}^{F}(t) & u_{2}^{F}(t) & \cdots & u_{m}^{F}(t)
\end{array}\right]^{T}, \\
& \breve{\alpha}=\operatorname{diag}\left\{\breve{\alpha}_{1}, \breve{\alpha}_{2}, \cdots, \breve{\alpha}_{m}\right\}, \\
& \widehat{\alpha}=\operatorname{diag}\left\{\hat{\alpha}_{1}, \hat{\alpha}_{2}, \cdots, \hat{\alpha}_{m}\right\} \text {, } \\
& \alpha=\operatorname{diag}\left\{\alpha_{1}, \alpha_{2}, \cdots, \alpha_{m}\right\} \text {, }
\end{aligned}
$$

$\alpha$ is said to be admissible if $\alpha$ satisfies $\hat{\alpha} \leq \alpha \leq \breve{\alpha}$.

The objective of this paper is to develop a procedure to design a memoryless state feedback control law

$$
u(t)=K x(t)
$$

such that for any admissible uncertain $\alpha$, the resulting closed-loop system

$$
\begin{aligned}
\dot{x}(t)= & \left(A+B \alpha K+D F E_{1}+D F E_{2} \alpha K\right) x(t) \\
& +\left(A_{d}+D F E_{d}\right) x(t-\tau(t))+f, \quad t \in R^{+}, \\
x(t)= & \phi(t), \quad t \in[-h, 0],
\end{aligned}
$$

is quadratically stable and the cost function (4) satisfies $J \leq J^{*}$, where $J^{*}$ is some specified constant.

Definition 1. If there exists a control $u(t)=K x(t)$ and a positive scalar $J^{*}$ such that for all admissible $\alpha$, the closed-loop system (9) is quadratically stable and $J \leq J^{*}$, then $J^{*}$ is said to be a guaranteed cost and $u(t)=K x(t)$ is 
said to be a reliable guaranteed cost control law for system (1) and cost function (4).

Define

$$
\beta=\operatorname{diag}\left\{\beta_{1}, \beta_{2}, \cdots, \beta_{m}\right\}, \quad \beta_{0}=\operatorname{diag}\left\{\beta_{10}, \beta_{20}, \cdots, \beta_{m 0}\right\},
$$

where

$$
\beta_{i}=\frac{\widehat{\alpha}_{i}+\breve{\alpha}_{i}}{2}, \quad \beta_{i 0}=\frac{\breve{\alpha}_{i}-\widehat{\alpha}_{i}}{\breve{\alpha}_{i}+\widehat{\alpha}_{i}}, \quad i=1,2, \cdots, m .
$$

From (7) and (10), we define

$$
\alpha=\left(I+\alpha_{0}\right) \beta
$$

and

$$
\left|\alpha_{0}\right| \leq \beta_{0} \leq I
$$

where $\quad \alpha_{0}=\operatorname{diag}\left\{\alpha_{01}, \alpha_{02}, \cdots, \alpha_{0 m}\right\} \quad, \quad$ and $\left|\alpha_{0}\right|=\operatorname{diag}\left\{\left|\alpha_{01}\right|,\left|\alpha_{02}\right|, \cdots,\left|\alpha_{0 m}\right|\right\}$.

Lemma 1. (Schur complement). Given the constant matrices $\Omega_{1}, \Omega_{2}, \Omega_{3}$ of appropriate dimensions, where $\Omega_{1}=\Omega_{1}^{T}$ and $\Omega_{2}=\Omega_{2}^{T}>0$, then

$$
\Omega_{1}+\Omega_{3}^{T} \Omega_{2}^{-1} \Omega_{3}<0
$$

if and only if

$$
\left[\begin{array}{cc}
\Omega_{1} & \Omega_{3}^{T} \\
\Omega_{3} & -\Omega_{2}
\end{array}\right]<0 \text { or }\left[\begin{array}{cc}
-\Omega_{2} & \Omega_{3} \\
\Omega_{3}^{T} & \Omega_{1}
\end{array}\right]<0 .
$$

Lemma 2. (Barmish [17]) Given matrices $Y, H, E$ of appropriate dimensions and with $Y$ symmetric, then for all $F$ satisfying $F^{T} F \leq I$ and

$$
Y+H F E+E^{T} F^{T} H^{T}<0,
$$

if and only if there exists $\varepsilon>0$ such that

$$
Y+\varepsilon H H^{T}+\varepsilon^{-1} E^{T} E<0 .
$$

Lemma 3. (Moon et al. [18]) Assume that $a \in R^{p}, b \in R^{q}$, and $N \in R^{p \times q}$, then for any matrices $X \in R^{p \times p}, Y \in R^{p \times q}$, $Z \in R^{q \times q}$, the following holds:

$$
-2 a^{T} N b \leq\left[\begin{array}{l}
a \\
b
\end{array}\right]^{T}\left[\begin{array}{cc}
X & Y-N \\
Y^{T}-N^{T} & Z
\end{array}\right]\left[\begin{array}{l}
a \\
b
\end{array}\right],
$$

if

$$
\left[\begin{array}{cc}
X & Y \\
Y^{T} & Z
\end{array}\right] \geq 0
$$

\section{Main Results}

Since it holds that

$$
x(t-\tau(t))=x(t)-\int_{t-\tau(t)}^{t} \dot{x}(s) d s .
$$

Then, rewrite system (9) in an equivalent form

$$
\begin{aligned}
& \dot{x}(t)=\left(A_{C}+A_{D}\right) x(t)-A_{D} \int_{t-\tau(t)}^{t} \dot{x}(s) d s+f, \quad t \in R^{+}, \\
& x(t)=\phi(t), \quad t \in[-h, 0],
\end{aligned}
$$

where,

$$
\begin{gathered}
A_{C}=A+B \alpha K+D F E_{1}+D F E_{2} \alpha K \\
A_{D}=A_{d}+D F E_{d} .
\end{gathered}
$$

The following Lyapunov-Krasovskii functional is applied

$$
V(t)=V_{1}(t)+V_{2}(t)+V_{3}(t),
$$

where

$$
\begin{gathered}
V_{1}(t)=x^{T}(t) \operatorname{Px}(t), \\
V_{2}(t)=\int_{-\tau(t)}^{0} \int_{t+\theta}^{t} \dot{x}^{T}(s) R \dot{x}(s) d s d \theta, \\
V_{3}(t)=\int_{t-\tau(t)}^{t} x^{T}(\tau) S x(\tau) d \tau .
\end{gathered}
$$

Then, the following theorem gives the delay-dependent reliable guaranteed cost control for the systems (1) and (4).

Theorem 1. $u(t)=K x(t)$ is a reliable guaranteed cost control law if there exist positive-definite matrices $P, S, R$, matrices $Y, Z$, and a scalar $\varepsilon_{1}>0$, such that for any admissible $\alpha$, the following matrix inequalities hold:

$$
\Psi=\left[\begin{array}{ccc}
\Psi_{11} & \Psi_{12} & \Psi_{13} \\
* & \Psi_{22} & \Psi_{23} \\
* & * & \Psi_{33}
\end{array}\right]<0,
$$

and

$$
\left[\begin{array}{ll}
Z & Y \\
* & R
\end{array}\right] \geq 0
$$

where $(*)$ denotes the symmetric element of a matrix, and 


$$
\begin{aligned}
& \Psi_{11}=A_{C}^{T} h R A_{C}+A_{C}^{T} P+P A_{C}+h Z+Y+Y^{T}+S+\varepsilon_{1}^{-1} H_{1}^{T} H_{1}+Q+K^{T} \alpha R \alpha K, \\
& \Psi_{12}=A_{C}^{T} h R A_{D}+P A_{D}-Y, \\
& \Psi_{13}=A_{C}^{T} h R+P, \\
& \Psi_{22}=-S(1-d)+\varepsilon_{1}^{-1} H_{2}^{T} H_{2}+A_{D}^{T} h R A_{D}, \\
& \Psi_{23}=A_{D}^{T} h R, \\
& \Psi_{33}=h R-\varepsilon_{1}^{-1} I,
\end{aligned}
$$

Moreover, the cost function (4) satisfies the following bound:

$$
J \leq x^{T}(0) P x(0)+\int_{-\tau(t)}^{0} \int_{\theta}^{0} \dot{x}^{T}(s) R \dot{x}(s) d s d \theta+\int_{-\tau(t)}^{0} x^{T}(\tau) S x(\tau) d \tau .
$$

Proof. Taking $u(t)=K x(t)$ in the system (1), the resulting closed-loop system is given by (9).

Differentiating $V_{1}(t)$ with respect to $t$ gives

$$
\begin{aligned}
\dot{V}_{1}(t) & =\dot{x}^{T}(t) P x(t)+x^{T}(t) P \dot{x}(t) \\
& =\left[\left(A_{C}+A_{D}\right) x(t)-A_{D} \int_{t-\tau(t)}^{t} \dot{x}(s) d s+f\right]^{T} P x(t)+x^{T}(t) P\left[\left(A_{C}+A_{D}\right) x(t)-A_{D} \int_{t-\tau(t)}^{t} \dot{x}(s) d s+f\right] \\
& =x^{T}(t)\left[\left(A_{C}+A_{D}\right)^{T} P+P\left(A_{C}+A_{D}\right)\right] x(t)-2 x^{T}(t) P A_{D} \int_{t-\tau(t)}^{t} \dot{x}(s) d s+x^{T}(t) P f+f^{T} P x(t)
\end{aligned}
$$

Taking

$$
N=P A_{D}, X=Z, Y=Y, Z=R, a=x(t), b=\dot{x}(s),
$$

we obtain

$$
\begin{aligned}
-2 x^{T}(t) P A_{D} \int_{t-\tau(t)}^{t} \dot{x}(s) d s= & -2 \int_{t-\tau(t)}^{t} x^{T}(t) P A_{D} \dot{x}(s) d s \\
& \leq \int_{t-\tau(t)}^{t}\left[\begin{array}{ll}
x^{T}(t) & \dot{x}^{T}(s)
\end{array}\right]\left[\begin{array}{cc}
Z & Y-P A_{D} \\
Y^{T}-A_{D}{ }^{T} P & R
\end{array}\right]\left[\begin{array}{c}
x(t) \\
\dot{x}(s)
\end{array}\right] d s \\
& \leq x^{T}(t)\left(Y^{T}-A_{D}^{T} P+Y-P A_{D}\right) x(t)+\int_{t-\tau(t)}^{t} \dot{x}^{T}(s) R \dot{x}(s) d s \\
& -x^{T}(t-\tau(t))\left(Y^{T}-A_{D}^{T} P\right) x(t)-x^{T}(t)\left(Y-P A_{D}\right) x(t-\tau(t))+h x^{T}(t) Z x(t)
\end{aligned}
$$

Substituting (25) into (23) and using (3), we have

$$
\begin{aligned}
\dot{V}_{1}(t) \leq & x^{T}(t)\left[A_{C}^{T} P+P A_{C}+Y^{T}+Y+h Z\right] x(t)+x^{T}(t) P f+f^{T} P x(t)-x^{T}(t)\left(Y-P A_{D}\right) x(t-\tau(t)) \\
& -x^{T}(t-\tau(t))\left(Y^{T}-A_{D}^{T} P\right) x(t)+\int_{t-\tau(t)}^{t} \dot{x}^{T}(s) R \dot{x}(s) d s-\varepsilon_{1}^{-1} f^{T} f+\varepsilon_{1}^{-1}\left[x^{T}(t) \quad x^{T}(t-\tau(t))\right] H\left[x^{T}(t) \quad x^{T}(t-\tau(t))\right]^{T}
\end{aligned}
$$

Differentiating $V_{2}(t)$ and $V_{3}(t)$ with respect to $t$ gives

$$
\begin{aligned}
\dot{V}_{2}(t) & =\int_{-\tau(t)}^{0}\left[\dot{x}^{T}(t) R \dot{x}(t)-\dot{x}^{T}(t+\theta) R \dot{x}(t+\theta)\right] d \theta=\tau(t) \dot{x}^{T}(t) R \dot{x}(t)-\int_{t-\tau(t)}^{t} \dot{x}^{T}(s) R \dot{x}(s) d s \\
& \leq h \dot{x}^{T}(t) R \dot{x}(t)-\int_{t-\tau(t)}^{t} \dot{x}^{T}(s) R \dot{x}(s) d s \\
& \leq h\left[A_{C} x(t)+A_{D} x(t-\tau(t))+f\right]^{T} R\left[A_{C} x(t)+A_{D} x(t-\tau(t))+f\right]-\int_{t-\tau(t)}^{t} \dot{x}^{T}(s) R \dot{x}(s) d s
\end{aligned}
$$




$$
\begin{aligned}
\dot{V}_{3}(t) & =x^{T}(t) S x(t)-(1-\dot{\tau}(t)) x^{T}(t-\tau(t)) S x(t-\tau(t)) \\
& \leq x^{T}(t) S x(t)-(1-d) x^{T}(t-\tau(t)) S x(t-\tau(t))
\end{aligned}
$$

From the above inequalities, we can obtain

$$
\begin{aligned}
\dot{V}(t) & \leq \dot{V}_{1}(t)+\dot{V}_{2}(t)+\dot{V}_{3}(t) \\
& \leq \zeta^{T}(t) \Psi \zeta(t)-x^{T}(t)\left[Q+K^{T} \alpha R \alpha K\right] x(t)
\end{aligned}
$$

where

$$
\zeta(t)=\operatorname{col}\{x(t) \quad x(t-\tau(t)) \quad f\} .
$$

Then, the matrix inequality (19) implies that

$$
\begin{aligned}
& \int_{0}^{T_{f}} x^{T}(t)\left[Q+K^{T} \alpha R \alpha K\right] x(t) d t<V(0)-V\left(T_{f}\right) \\
= & x^{T}(0) \operatorname{Px}(0)+x^{T}\left(T_{f}\right) \operatorname{Px}\left(T_{f}\right)+\int_{-\tau(t)}^{0} \int_{\theta}^{0} \dot{x}^{T}(s) R \dot{x}(s) d s d \theta+\int_{-\tau(t)}^{0} x^{T}(\tau) S x(\tau) d \tau \\
& +\int_{-\tau(t)}^{0} \int_{T_{f}+\theta}^{T_{f}} \dot{x}^{T}(s) R \dot{x}(s) d s d \theta+\int_{T_{f}-\tau(t)}^{T_{f}} x^{T}(\tau) S x(\tau) d \tau .
\end{aligned}
$$

As the closed-loop systems (9) is asymptotically stable when $T_{f} \rightarrow \infty$, we get

$$
\int_{0}^{\infty}\left(x^{T}(t) Q x(t)+u^{T}(t) R u(t)\right) d t \leq V(0)=J^{*} .
$$

$$
\begin{aligned}
& x^{T}\left(T_{f}\right) \operatorname{Px}\left(T_{f}\right) \rightarrow 0, \\
& \int_{-\tau(t)}^{0} \int_{T_{f}+\theta}^{T_{f}} \dot{x}^{T}(s) R \dot{x}(s) d s d \theta \rightarrow 0, \\
& \int_{T_{f}-\tau(t)}^{T_{f}} x^{T}(\tau) S x(\tau) d \tau \rightarrow 0 .
\end{aligned}
$$

Hence, we get
This completes the proof.

In the sequel, we will show that the criterion for the existence of guaranteed cost controller is equivalent to the feasibility of a LMI.

Theorem 2. For system (1) with cost function (4), if there exist scalars $\varepsilon_{1}>0, \varepsilon_{2}>0$, matrices $X>0, Z>0$, $M>0, R_{0}>0$, such that the following LMI is feasible:

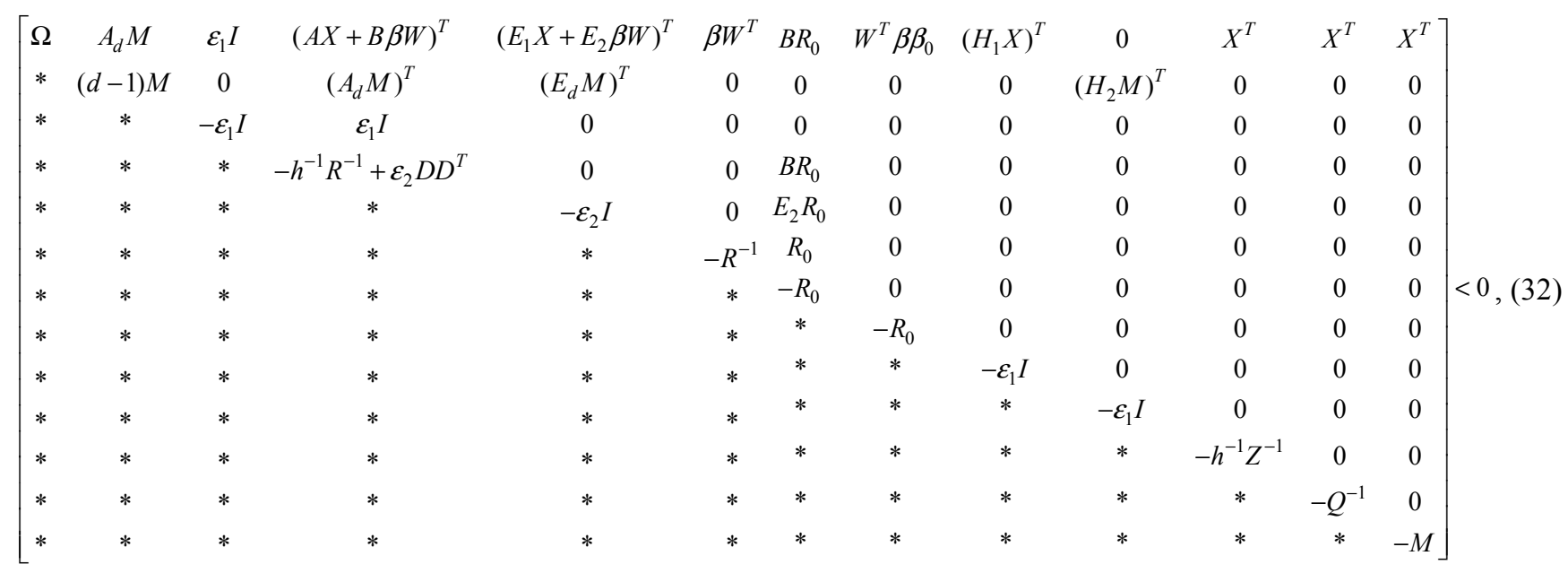

where

$$
\begin{aligned}
& \Omega=(A X+B W)^{T}+A X+B W+\varepsilon_{2} D D^{T}, \\
& X=P^{-1}, \quad W=K P^{-1}, \quad M=S^{-1},
\end{aligned}
$$

then 


$$
u(k)=W X^{-1} x(k)
$$

is a reliable guaranteed cost controller of system (1), and the corresponding closed-loop value of the cost function satisfies

$$
J \leq x^{T}(0) X^{-1} x(0)+\int_{-\tau(t)}^{0} \int_{\theta}^{0} \dot{x}^{T}(s) R \dot{x}(s) d s d \theta+\int_{-\tau(t)}^{0} x^{T}(\tau) M^{-1} x(\tau) d \tau .
$$

Proof. Letting $Y \equiv 0$ and $Z>0$ in (20), in light of Lemma 2 and using the Schur complement, we can obtain

$$
\left[\begin{array}{cccc}
\Psi_{11}-A_{C}^{T} h R A_{C} & P A_{D} & P & A_{C}^{T} \\
* & -S(1-d)+\varepsilon_{1}^{-1} H_{2}^{T} H_{2} & 0 & A_{D}^{T} \\
* & * & -\varepsilon_{1}^{-1} I & I \\
* & * & * & -h^{-1} R^{-1}
\end{array}\right]<0
$$

substituting the representation of matrix $A_{C}, A_{D}$ into (35), and using the Schur complement again, we can obtain

$$
\left[\begin{array}{ccccc}
\Gamma & P A_{d} & P & (A+B \alpha K)^{T} & \left(E_{1}+E_{2} \alpha K\right)^{T} \\
* & -S(1-d)+\varepsilon_{1}^{-1} H_{2}^{T} H_{2} & 0 & A_{d}^{T} & E_{d}^{T} \\
* & * & -\varepsilon_{1}^{-1} I & I & 0 \\
* & * & * & -h^{-1} R^{-1}+\varepsilon_{2} D D^{T} & 0 \\
* & * & * & * & -\varepsilon_{2} I
\end{array}\right]<0
$$

where,

$$
\Gamma=(A+B \alpha K)^{T} P+P(A+B \alpha K)+h Z+S+\varepsilon_{1}^{-1} H_{1}^{T} H_{1}+Q+K^{T} \alpha R \alpha K+\varepsilon_{2} P D D^{T} P .
$$

Using the Schur complement again, and by (12), (13), we can get

$$
\begin{aligned}
& {\left[\begin{array}{ccc}
\Gamma_{1} & P A_{d} & P \\
* & -S(1-d)+\varepsilon_{1}^{-1} H_{2}^{T} H_{2} & 0 \\
* & * & -\varepsilon_{1}^{-1} I \\
* & * & * \\
* & * & * \\
* & * & *
\end{array}\right.} \\
& \left.\begin{array}{ccc}
(A+B \beta K)^{T} & \left(E_{1}+E_{2} \beta K\right)^{T} & K^{T} \beta \\
A_{d}^{T} & E_{d}^{T} & 0 \\
I & 0 & 0 \\
-h^{-1} R^{-1}+\varepsilon_{2} D D^{T} & 0 & 0 \\
* & -\varepsilon_{2} I & 0 \\
* & * & -R^{-1}
\end{array}\right] \\
& +\left[\begin{array}{c}
P B \\
0 \\
0 \\
B \\
E_{2} \\
I
\end{array}\right] \alpha_{0}\left[\begin{array}{llllll}
\beta K & 0 & 0 & 0 & 0 & 0
\end{array}\right]+\left(\left[\begin{array}{c}
P B \\
0 \\
0 \\
B \\
E_{2} \\
I
\end{array}\right] \alpha_{0}\left[\begin{array}{llllll}
\beta K & 0 & 0 & 0 & 0 & 0
\end{array}\right]\right)^{T}<0,
\end{aligned}
$$

where

$$
\Gamma_{1}=(A+B \beta K)^{T} P+P(A+B \beta K)+h Z+S+\varepsilon_{1}^{-1} H_{1}^{T} H_{1}+Q+\varepsilon_{2} P D D^{T} P .
$$

Using the inequality $2 a^{T} b \leq a^{T} a+b^{T} b$ for any diagonal matrix $R_{0}>0$, it follows that 


$$
\left[\begin{array}{cccccccc}
\Gamma_{1} & P A_{d} & P & (A+B \beta K)^{T} & \left(E_{1}+E_{2} \beta K\right)^{T} & K^{T} \beta & P B & K^{T} \beta \beta_{0} \\
* & -S(1-d)+\varepsilon_{1}^{-1} H_{2}^{T} H_{2} & 0 & A_{d}^{T} & E_{d}^{T} & 0 & 0 & 0 \\
* & * & -\varepsilon_{1}^{-1} I & I & 0 & 0 & 0 & 0 \\
* & * & * & -h^{-1} R^{-1}+\varepsilon_{2} D D^{T} & 0 & 0 & B & 0 \\
* & * & * & * & -\varepsilon_{2} I & 0 & E_{2} & 0 \\
* & * & * & * & * & -R^{-1} & I & 0 \\
* & * & * & * & * & * & -R_{0}^{-1} & 0 \\
* & * & * & * & * & * & * & -R_{0}
\end{array}\right]<0
$$

Pre- and post-multiplying both sides of the above inequality by $\Pi^{T}$ and $\Pi$, where

$$
\Pi=\operatorname{diag}\left\{P^{-1}, S^{-1}, \varepsilon_{1} I, I, I, I, R_{0}, I\right\},
$$

we can obtain (32). This completes the proof.

Theorem 2 presents a method of designing a state feedback reliable guaranteed cost controller. The following theorem presents a method of selecting a controller minimizing the upper bound of the guaranteed cost (34).

Theorem 3. Consider the systems (1) with performance index (4), if the following optimization problem

$$
\min \xi+\operatorname{tr}\left(\Gamma_{1}\right)+\operatorname{tr}\left(\Gamma_{2}\right)
$$

(i) (32),

$$
\begin{aligned}
& \text { (ii) }\left[\begin{array}{cc}
-\xi & x^{T}(0) \\
x(0) & -X
\end{array}\right]<0, \\
& \text { (iii) }\left[\begin{array}{cc}
-\Gamma_{1} & C^{T} \\
C & -R^{-1}
\end{array}\right]<0,
\end{aligned}
$$

(iv) $\left[\begin{array}{cc}-\Gamma_{2} & D^{T} \\ D & -M\end{array}\right]<0$,

has a solution set $\left(X, \xi, \Gamma_{1}, \Gamma_{2}\right)$, then the controller (33) is an optimal reliable guaranteed cost control law which ensures the minimization of the guaranteed cost (34) for system (1), where

$$
\begin{aligned}
& \int_{-\tau(t)}^{0} \int_{\theta}^{0} \dot{x}(s) \dot{x}^{T}(s) d s d \theta=C^{T} C, \\
& \int_{-\tau(t)}^{0} x(\tau) x^{T}(\tau) d \tau=D^{T} D
\end{aligned}
$$

Proof. By Theorem 2, (i) in (37) is clear. It follows Lemma 1 that (ii)-(iv) in (37) are equivalent to

$$
x^{T}(0) X^{-1} x(0)<\xi, \quad C^{T} R C<\Gamma_{1}, D^{T} M^{-1} D<\Gamma_{2},
$$

respectively. Furthermore,

$$
\begin{aligned}
\int_{-\tau(t)}^{0} \int_{\theta}^{0} \dot{x}^{T}(s) R \dot{x}(s) d s d \theta & =\int_{-\tau(t)}^{0} \int_{\theta}^{0} \operatorname{tr}\left(\dot{x}^{T}(s) R \dot{x}(s)\right) d s d \theta \\
& =\operatorname{tr}\left(C^{T} R C\right)<\operatorname{tr}\left(\Gamma_{1}\right)
\end{aligned}
$$

and

$$
\begin{aligned}
& \int_{-\tau(t)}^{0} x^{T}(\tau) M^{-1} x(\tau) d \tau \\
= & \int_{-\tau(t)}^{0} \operatorname{tr}\left(x^{T}(\tau) M^{-1} x(\tau)\right) d \tau . \\
= & \operatorname{tr}\left(D^{T} M^{-1} D\right)<\operatorname{tr}\left(\Gamma_{2}\right)
\end{aligned}
$$

Hence, it follows from (34) that

$$
\begin{aligned}
J^{*} & =x^{T}(0) X^{-1} x(0)+\int_{-\tau(t)}^{0} \int_{\theta}^{0} \dot{x}^{T}(s) R \dot{x}(s) d s d \theta+\int_{-\tau(t)}^{0} x^{T}(\tau) M^{-1} x(\tau) d \tau \\
& \leq \xi+\operatorname{tr}\left(\Gamma_{1}\right)+\operatorname{tr}\left(\Gamma_{2}\right)
\end{aligned}
$$

Thus, the minimization of (42) implies the minimization of the guaranteed cost for the system (1). The optimality of the solutions of the optimization problem (37) follows from the convexity of the objective function and of the constraints, which ensures that a global optimum, when it exists, is reachable. This completes the proof. 


\section{Simulations}

Consider the systems with time-varying delay in (1)-(4) with

$$
\begin{aligned}
& A=\left[\begin{array}{ll}
0 & 1 \\
9 & 0
\end{array}\right], \quad B=\left[\begin{array}{l}
3 \\
1
\end{array}\right], \quad A_{d}=\left[\begin{array}{ll}
0.5 & 0.3 \\
0.3 & 0.5
\end{array}\right], \quad D=\left[\begin{array}{ccc}
0.1 & 0 & 0.1 \\
0 & 0.1 & 0
\end{array}\right], \\
& E_{1}=\left[\begin{array}{ll}
1 & 1 \\
0 & 0 \\
0 & 0
\end{array}\right], E_{2}=\left[\begin{array}{l}
0 \\
1 \\
0
\end{array}\right], E_{d}=\left[\begin{array}{ll}
0 & 0 \\
0 & 0 \\
1 & 1
\end{array}\right], H_{1}=H_{2}=\left[\begin{array}{cc}
0.1 & 0 \\
0 & 0.1
\end{array}\right] \text {, } \\
& Q=\operatorname{diag}\{1,1\}, \quad R=0.2, \quad h=1, \quad d=0.5, \quad Z=\left[\begin{array}{cc}
0.1 & 0 \\
0 & 0.1
\end{array}\right] .
\end{aligned}
$$

It is assumed that the single input to the system has partial failure as follows:

$$
\widehat{\alpha}=0.8, \breve{\alpha}=1.2 \text {. }
$$

By applying Theorem 3 and solving the corresponding optimization problem (37), the optimal solution is given by

$$
\begin{gathered}
X=\left[\begin{array}{cc}
0.2620 & -0.3360 \\
-0.3360 & 0.9191
\end{array}\right], W=\left[\begin{array}{ll}
-0.7521 & -0.2579
\end{array}\right], \xi=53.1304, \\
\Gamma_{1}=\left[\begin{array}{cc}
0.6677 & 0 \\
0 & 0.0288
\end{array}\right], \quad \Gamma_{2}=\left[\begin{array}{cc}
11.3919 & 0 \\
0 & 0.0288
\end{array}\right] .
\end{gathered}
$$

Then, the optimal reliable guaranteed cost controller is given by

$$
u(k)=\left[\begin{array}{ll}
-6.0845 & -2.5053
\end{array}\right] x(k),
$$

and the upper bound of the corresponding closed-loop cost function is $J^{*}=65.2476$.

\section{Conclusions}

In this paper, based on the Lyapunov method, we have presented a design method to the reliable guaranteed cost controller via memoryless state feedback control for uncertain systems with time-varying state delay and nonlinear perturbations in an LMI framework. The parameterized representation of a set of the controller, which guaranteed not only the robust stability of the closed-loop system but also the cost function bound constraint, has been provided in terms of the feasible solutions to the LMIs. Furthermore, a convex optimization problem has been introduced to select the optimal reliable guaranteed cost controller. Finally, a numerical example is given for illustration of the controller design.

\section{References}

[1] W. P. Lu, H. D. Xu, S. Y. Li, et al. Reliable guaranteed cost Control for uncertain time-lag systems of actuator failures [J]. Key Engineering Materials, 2010, 930: 960-965.

[2] I. Petersen, D. McFarlane. Optimal guaranteed cost control and filtering for uncertain linear systems [J]. IEEE Trans. Automat. Control, 1994, 39 (9): 1971-1977.

[3] N. Xie, G. Y. Tang, Delay-dependent nonfragile guaranteed cost control for nonlinear time-delay systems [J]. Nonlinear Analysis, 2006, 64 (9): 2084-2097.

[4] L. Yu, F. Gao, Optimal guaranteed cost control of discrete-time uncertain systems with both state and input delays [J]. Journal of the Franklin Institute, 2001, 338 (1): 101-110.

[5] Y. G. Kao, J. Xie, C. H. Wang. A sliding mode approach to $\mathrm{H} \infty$ non-fragile observer-based control design for uncertain markovian neutral-type stochastic systems [J]. Automation, 2015, 52: 218-226.

[6] Z. P. Yin, K. Yu, Y. Wang. Robust control algorithm and simulation of networked control systems. Computer Communications, 2020, 157: 394-401.

[7] Q. X. Zhu, K. H. Lu, Y. H. Zhu, S. S. Hu. The guaranteed-cost controller design for networked-control system with markov delay [J]. Aerospace Control, 2014, 32 (04): 3-7.

[8] J. Song. Fault-Tolerant control of networked control systems with time-varying actuator fault [J]. Aerospace Control, 2016, 34 (05): 3-8.

[9] X. Z. Liu. Research on fault toleran control of networked control systems [D]. Beijing institute of technology, 2016.

[10] Q. Zhu, K. Lu, G. M. Xie, et al. Guaranteed cost fault-tolerant control for networked control systems with sensor faults [J]. Mathematical Problems in Engineering, 2015, 2015.

[11] P. P. Yao, X. J. Fu. Reliable guaranteed cost control for a class of nonlinear uncertain time-delay systems [J]. Computing Technology and Automation, 2017, 36 (01): 1-5. 
[12] S. Deng, L. C. Yang. Reliable Ho control design of discrete-time Takagi-Sugeno fuzzy systems with actuator faults [J]. Neurocomputing, 2016, 173 (3): 1784-1788.

[13] J. S. Zhang, Y. W. Wang, J. W. Xiao, et al. Robust reliable guaranteed cost control of positive interval systems with multiple time delays and actuator failure [J]. International Journal of Systems Science, 2016, 47 (4): 946-955.

[14] Q. X. Zhu, K. H. Lu, Y. H. Zhu. Guaranteed cost fault-tolerant control of double-fault networked control systems: piecewise delay method [J]. Mathematical Problems in Engineering, 2019, (9): 1-19.

[15] S. X. SUN, et al. Reliable Hळ guaranteed cost control for uncertain switched fuzzy stochastic systems with multiple time-varying delays and intermittent actuator and sensor faults. Neural Computing and Applications, 2020, (4): 1-23.

[16] S. Q. Zou, H. Yan, L. Zhang. Reliable static output feedback guaranteed cost control for uncertain systems with time-delay [C]. In: Chinese Intelligent Automation Conference. Springer, Singapore, 2017, 458: 407-413.

[17] B. R. Barmish. Necessary and sufficient conditions for quadratic stability of an uncertain system [J]. Journal of Optimization Theory and Applications, 1985, 46: 399-408.

[18] Y. S. Moon, P. Park, W. H. Kwon, Y. S. Lee. Delay-dependent robust stabilization of uncertain state-delayed systems [J], International Journal of Control, 2001, 74 (14): 1447-1455. 